\title{
STRUCTURE AND PROPERTIES OF METALS
}

\author{
K.J. KURZYDLOWSKI \\ Department of Materials Science and Engineering, Warsaw University of Technology \\ Narbutta 85,"02-524 Warszawa, Poland
}

\begin{abstract}
Metals are one of the most widely used types of engineering materials. Some of their properties, e.g. elastic constants, can be directly related to the nature of the metallic bonds between the atoms. On the other hand, macroand microstructural features of metals, such as point defects, dislocations, grain boundaries, and second phase particles, control their yield, flow, and fracture stress. Images of microstructural elements can be obtained by modern imaging techniques. Modern computer aided methods can be further used to obtain a quantitative description of these microstructures. These methods take advantage of the progress made in recent years in the field of image processing, mathematical morphology and quantitative stereology. Quantitative description of the microstructures are used for modeling processes taking place under the action of applied load at a given temperature and test (service) environment. These model considerations can be illustrated on the example of an anstenitic stainless steel, which is an important material for power generating and chemical industry. Reports recently published also show that properties of materials can be significantly modified by the effect of free surface. Examples of such situations include environmental effect on the mechanical properties of materials. Data for an austenitic stainless steel is used to discuss contribution of the free surface to the mechanical properties of metals.
\end{abstract}

PACS numbers: $62.20 .-\mathrm{x}, 62.20 . \mathrm{Fe}$

\section{Introduction}

Metals are one of the most important types of the materials. They have relatively high values of elastic constants and can be made strong by alloying and proper heat treatment. What is particularly important they combine stiffness and high strength with considerable ductility. As a result, most metals yield before they fracture and this makes them the most widely used engineering material (see Table I and for more information [1]).

$\mathrm{X}$-ray diffraction analysis shows that the atoms in metals usually are arranged in a regular, repeated three-dimensional pattern. The concept of the perfect 
TABLE I

Standard mechanical and physical properties of metals, ceramics, and polymers.

\begin{tabular}{l|c|c|c}
\hline \hline \multirow{2}{*}{ Parameter } & \multicolumn{3}{|c}{ Range of typical values } \\
\cline { 2 - 4 } & Metals & Ceramics & Polymers \\
\hline Density $\left[\mathrm{Mg} / \mathrm{m}^{3}\right]$ & $3-10$ & $2-4$ & $1-2$ \\
Elastic modulus $[\mathrm{GPa}]$ & $20-200$ & $100-1000$ & $0.1-10$ \\
Strength [MPa] & $20-2000$ & $1000-10000$ & $5-100$ \\
Fracture toughness $\left[\mathrm{MPa} \mathrm{m}{ }^{1 / 2}\right]$ & $10-200$ & $2-15$ & $0.5-10$ \\
Thermal conductivity $[\mathrm{W} / \mathrm{mK}]$ & $50-300$ & $1-100$ & $0.1-1$ \\
Thermal expansion $\left[10^{-6} / \mathrm{K}\right]$ & $5-30$ & $2-20$ & $20-500$ \\
Relative cost & $\mathrm{A}$ & $10 \mathrm{~A}$ & $0.3 \mathrm{~A}$
\end{tabular}

TABLE II

Examples of structure insensitive properties of metals.

\begin{tabular}{l|l}
\hline \multicolumn{1}{c|}{ Properties } & \multicolumn{1}{c}{ Parameters } \\
\hline Elastic properties & Elastic constants \\
& $\left(E, G, K, \nu, c_{11}, \ldots, s_{11}, \ldots\right)$ \\
Melting point & Melting temperature, $T_{\mathrm{m}}$ \\
Density & $\rho$ \\
Specific heat & $c_{v}$ \\
Coefficient of thermal expansion & $i$
\end{tabular}

TABLE III

Elements of lattice imperfections in crystalline materials.

\begin{tabular}{|c|c|c|c|}
\hline 0-dimensional & 1-dimensional & 2-dimensional & 3-dimensional \\
\hline $\begin{array}{l}\text { Vacancies, } \\
\text { impurities, } \\
\text { alloying atoms }\end{array}$ & Dislocations & $\begin{array}{l}\text { Stacking faults, } \\
\text { grain boundaries, } \\
\text { free surfaces, } \\
\text { interfaces, cracks }\end{array}$ & $\begin{array}{l}\text { Domains, grains, } \\
\text { aggregates, } \\
\text { second-phase } \\
\text { particles, pores }\end{array}$ \\
\hline
\end{tabular}

lattice of metal crystals can be used to explain the structure insensitive properties which are listed in Table II (see also [2]). The values of structure insensitive properties are primarily controlled by the strength of inter-atomic bonds, which in turn are influenced by the arrangements of atoms in crystal lattices.

Real metals deviate from the perfect periodicity of their mathematical models in the form of ideal crystals (see for example [3-6]).Various imperfections in 


\section{TABLE IV}

Examples of structure sensitive properties of metals.

\begin{tabular}{l|l}
\hline \multicolumn{1}{c|}{ Properties } & \multicolumn{1}{|c}{ Parameters } \\
\hline Yield stress & $\sigma_{y}, \sigma_{0,2}$ \\
Fracture strength & $\sigma_{\mathrm{f}}$, UTS \\
Hardness & $\mathrm{HV}, \mathrm{HB}$ \\
Creep resistance & $\sigma_{2}(100000)$
\end{tabular}

ideal arrangements of the atoms in crystalline materials have been divided, on the basis of their dimension, into the following classes:
a) point - 0-dimensional
b) linear - 1-dimensional,
c) surface - 2-dimensional,
d) volume - 3-dimensional.

Examples of the defects falling in these respective categories (a) to (d) are given in Table III. A number of useful properties of metals (see Table IV), are controlled by these imperfections which in turn are determined by its processing route and service conditions.

\section{Quantitative characterization of structure}

Images of the elements of microstructure of metals can be obtained with required precision by modern imaging techniques such as transmission electron microscopy (TEM), scanning electron microscopy (SEM), secondary-ion mass spectroscopy (SIMS), X-ray photoelectron spectroscopy (XPS). Examples of applications of these techniques are shown in Fig. 1. Modern computer aided methods can be further used to obtain a quantitative description of the microstructure of a studied material. These methods take advantage of the progress made in recent years in the field of image processing, mathematical morphology, and quantitative stereology.

Characterization of the microstructure of materials involves identification of the main microstructural elements present and a quantitative description of their sizes, shapes, numbers and positions within the specimen of the studied material. In other words this is a process that answers the following questions [7]:

1. what are the elements in the internal structure of a given material that distinguish it from other materials of that kind (of say similar chemical composition);

2. where are these elements located and in what quantity;

3. what is their size and shape?

Answers to all these questions provide a comprehensive description of the material microstructure that can be used to explain its properties and to gain better control over its technological usage. However, the first of these questions is a domain of material physics. In fact, microstructural elements such as dislocations and grain 

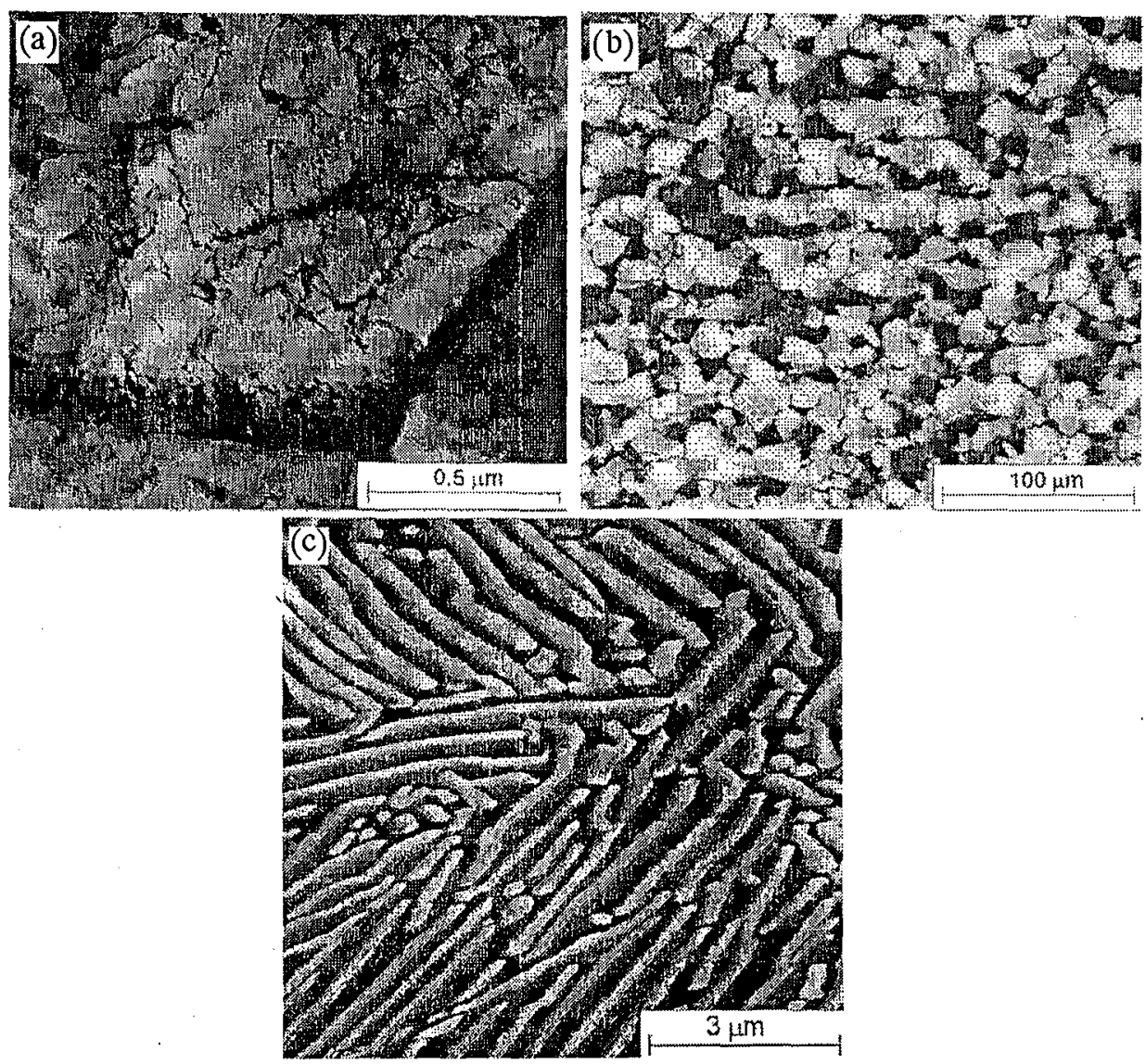

Fig. 1. Images of microstructural elements in metals: (a) dislocations and grain boundaries imaged by transmission electron microscopy; (b) grain and inter-phase boundaries imaged by light optical microscopy; (c) second phase particles revealed by scanning electron microscopy.

boundaries by themselves have become subjects of extensive theoretical studies. In the present text the focus is placed on the two last questions.

Size and shape are the attributes of 1-, 2- and 3-dimensional elements of the microstructure. The size of microstructural elements can be quantitatively described by:

- length, $l$, for 1-dimensional elements,

- area, $A$, for 2-dimensional elements,

- volume, $V$, for 3 -dimensional elements.

Direct measurements of the length, area, or volume of microstructural elements are rarely possible. As a result, the measurements are usually done on sections 
or projections of the microstructural elements. The results of such measurements characterize the size of the elements studied but do not define their volume, area, or length.

\section{Stereology}

The elements of a microstructure extend into 3 dimensions and are distributed over the volume of the specimen. This means that characterization of the microstructural elements should be based on some 3-dimensional model for the material studied. On the other hand, in an experimental approach they are commonly studied on 2-dimensional cross-sections or via examination of thin slices. On the other hand, a large number of properties are related to microstructural elements which are distributed over the volume of the material. In this situation, the required 3-dimensional description of the microstructure is inferred from the 2-dimensional images by means of the methods of quantitative stereology.

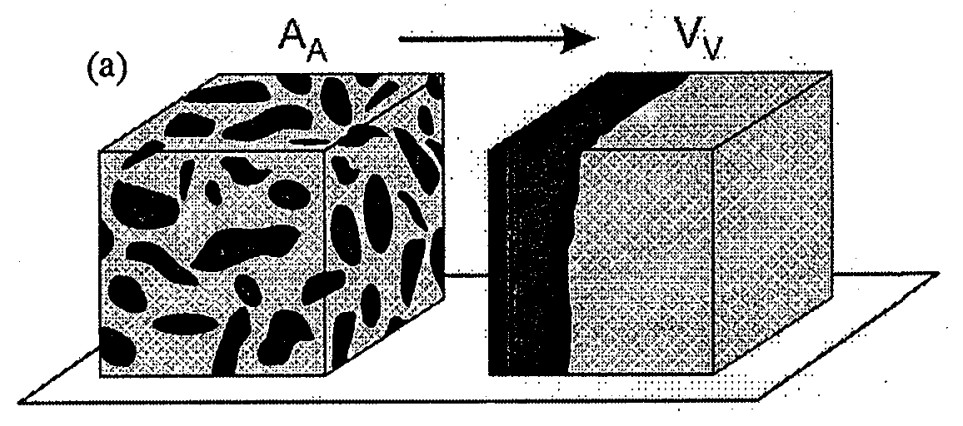

(b)

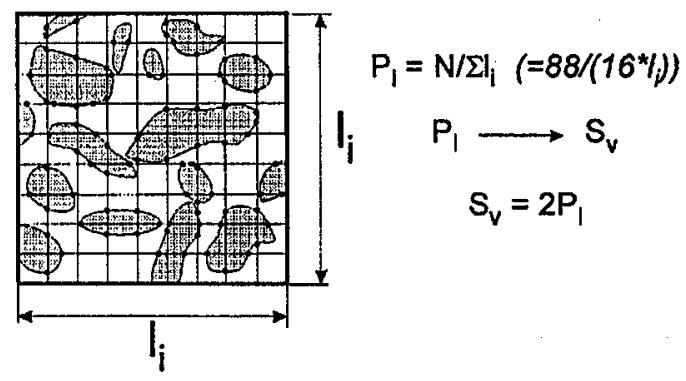

Fig. 2. Examples illustrating applications of stereological methods: (a) estimation of volume fraction, $V_{V}$, for a two-phase system via measurements of area fraction, $A_{A}$; (b) estimation of grain boundary surface are, $S_{V}$, via measurements of the density of intersection points for a system of test lines with traces of grain boundaries.

A quantitative description of the properties of two-dimensional images of microstructures is a prerequisite for the quantitative inference of the properties of the 3 -dimensional microstructure. In the past, a number of methods have been developed that allow an appropriate quantitative characterization of two-dimensional images by means of simple counting methods. Some details of such methods are given in Fig. 2. In recent years significant progress has been made in developing automatic computer-aided procedures (see Fig. 3). 


\section{The influence of microstructure on the properties of materials}

One of the foundations of modern materials science is the recognition of the fact that the properties of materials are related to their microstructure. For example, the properties of polycrystalline materials depend on the properties of grain boundaries and grain interiors, and on the amount of grain boundaries in a unit volume and, in turn, on tlie size of grains making up the polycrystalline aggregate. The grain size can be described and measured in different ways. The simplest procedure, frequently employed in physical metallurgy, is based on the concept of intercept measurements, schematically explained in Fig. 3. A system of test lines is superimposed on the image of the grain boundary network revealed on a cross-section of the polycrystal. The mean intercept length, $l$, is then calculated from a simple formula

$$
l=\frac{L_{\mathrm{T}}}{N_{i}},
$$

where $L_{\mathrm{T}}$ is the total length of the test lines and $N_{i}$ - the total number of their intersections with the traces of grain boundaries on the section. The value of $l$ decreases with decreasing grain size and the parameter is assumed to be a desirable measure of the grain size and as such is correlated with the grain boundary-sensitive properties of polycrystals.
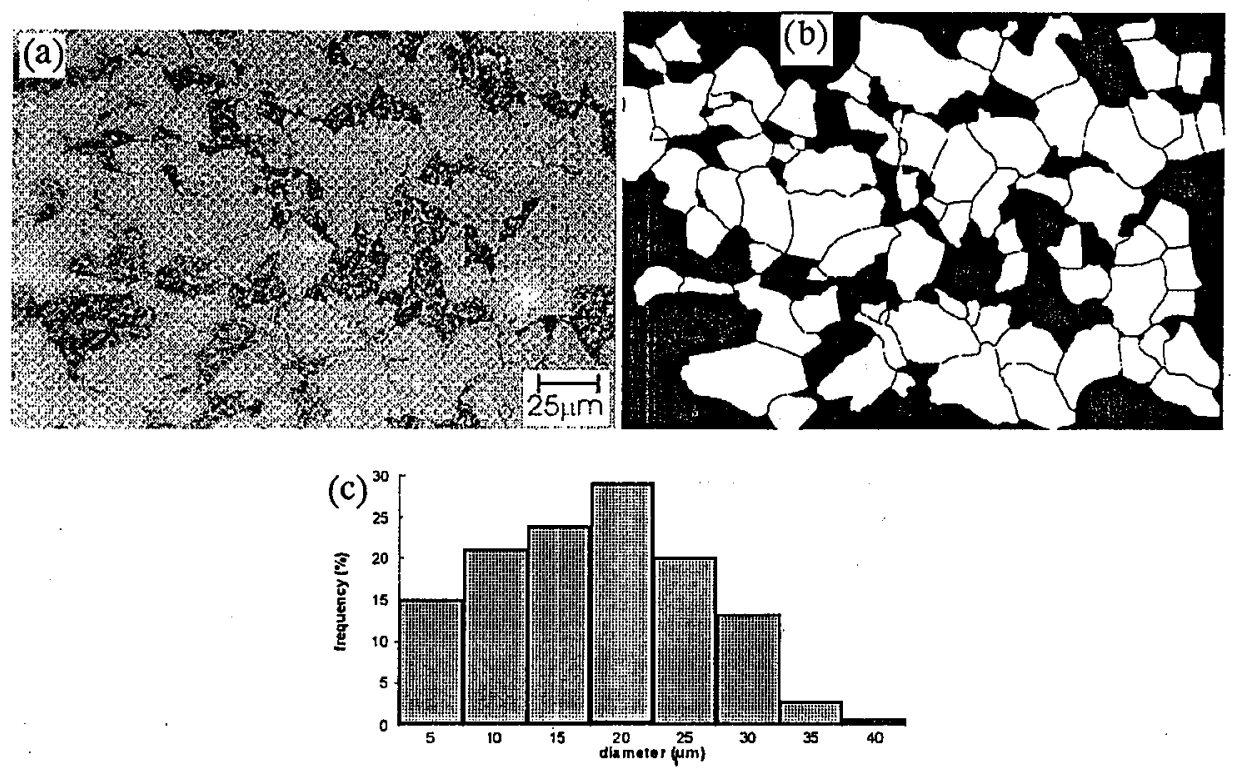

Fig. 3. Live image of a section of polycrystalline metals (a); its binary form revealing traces of grain boundaries (b) and histogram of grain section area $A$ (c).

Resistance to plastic deformation is one of the grain size-sensitive properties of polycrystals. One of the simple measures of this resistance is the hardness test. Some hardness tests, such as Vickers and Brinell, yield a number that defines the resistance to plastic deformation in MPa. Figure 4 presents the results of Vickers 


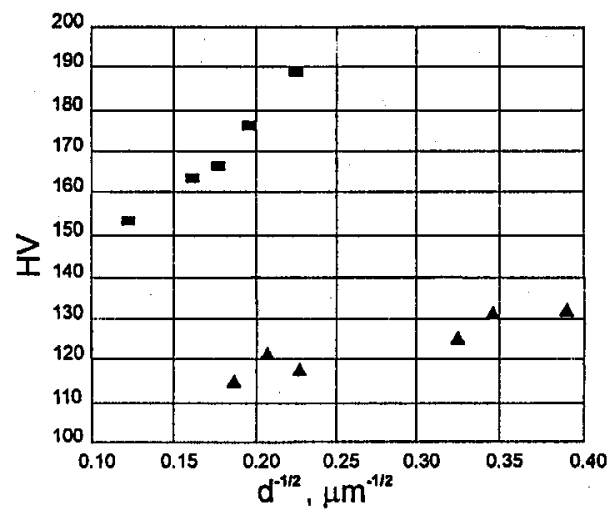

Fig. 4. The results of Vickers tests carried out on a series of specimens of a single-phase fcc alloy with different grain sizes (for details see [10]).

tests carried out on a series of specimens of a single-phase fcc alloy with different grain sizes. The hardness is plotted against $l^{-1 / 2}$ in accordance with a linear relationship

$$
H=H_{0}+K_{\mathrm{H}} l^{-1 / 2} \text {. }
$$

This formula, known as the Hall-Petch relationship [8-10], is one of the most useful in the field of physical metallurgy. It can be used to predict the properties of a wide range of materials including metals, ceramic, intermetallics and polymers.

\section{Free surface effect}

Reports recently published also show that properties of materials can be significantly modified by the effect of free surface. Examples of such situations include environmental effect on the mechanical properties of materials. Data for an austenitic stainless steel is used to discuss contribution of the free surface to the mechanical properties of metals.

In the temperature range from 200 to $700^{\circ} \mathrm{C}$ type 316 austenitic stainless steels exhibit a weak or a negative dependence of the macroscopic flow stress at a given total elongation, $\varepsilon$, on the test temperature, $T_{\mathrm{a}}$. In the literature such properties are related to dynamic strain aging (DSA). Part of this temperature interval is characterized by the presence of serrations on stress-strain curves. This phenomenon is termed as the Portevin-le Chatelier effect (PLC).

The PLC effect is generally linked to the interactions of dislocations with point defects. In the case of serrated flow in austenitic stainless steels interactions of carbon and chromium atoms, which form chromium carbides, were suggested to be relevant mechanism [11].

The environmental effect on the dynamic strain aging of 316 austenitic stainless steels is exemplified by the date presented in Fig. 5 . The specimens deformed. in the range of temperatures of the PLC effect in vacuum exhibit a lower frequency of serrations, higher values of the flow stress and higher ultimate tensile strength (UTS). 


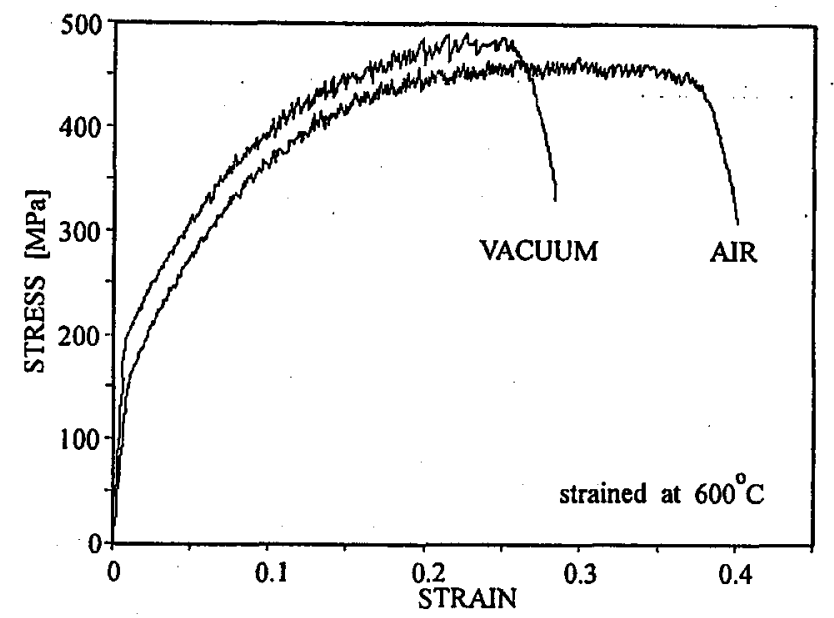

Fig. 5. The environmental effect on the dynamic strain aging of 316 austenitic stainless steels observed in static tensile straining.

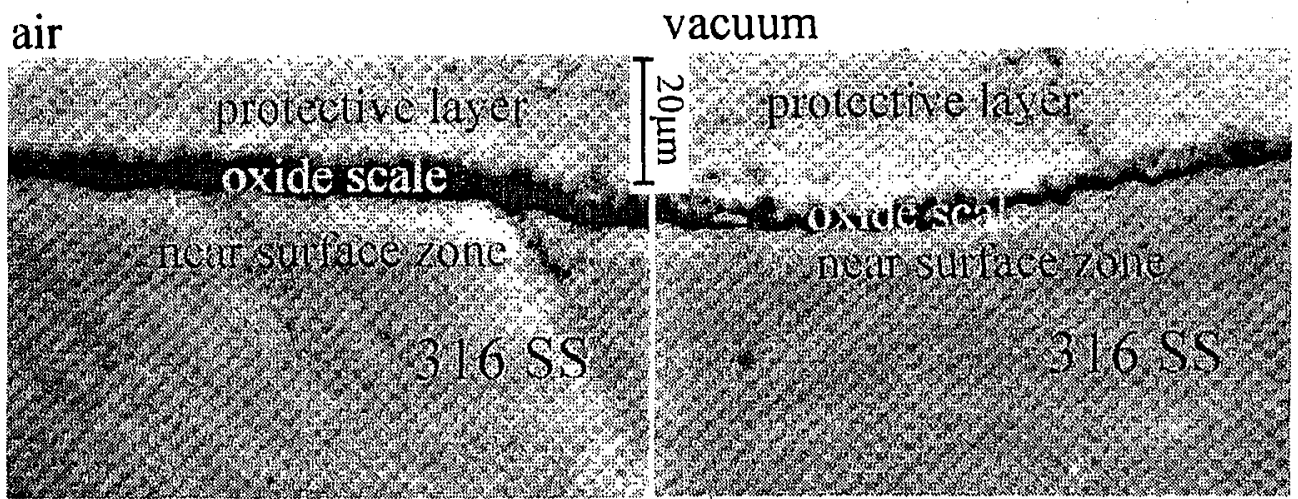

Fig. 6. Characteristic features of the scale formed as a result of annealing and straining in air and vacuum.

Observations of the outer surface of strained specimens were carried out using light and electron microscopy. Characteristic features of the scale formed as a result of annealing and straining in air and vacuum are illustrated by the micrographs shown in Fig. 6. Direct observations of the scale reveal a system of cracks nearly perpendicular to the axis of straining. The cracks, which are observed on the surface of specimens tested in air, are deeper, more regular and. more clearly elongated. Observations of the sections of the oxide scale show that it is characterized by relatively high roughness. The mean thickness of the scale depends on the test environment and temperature. In the case of straining at $600^{\circ} \mathrm{C}$ in air it exceeds $0.01 \mathrm{~mm}$ while for straining in air the mean thickness is smaller than $0.005 \mathrm{~mm}$.

More detailed examinations show that the oxide scale contains a stack of geometrically irregular layers containing a mixture of $\mathrm{FeO}, \mathrm{NiO}$ and $\mathrm{Cr}_{2} \mathrm{O}_{3}$. Outer 


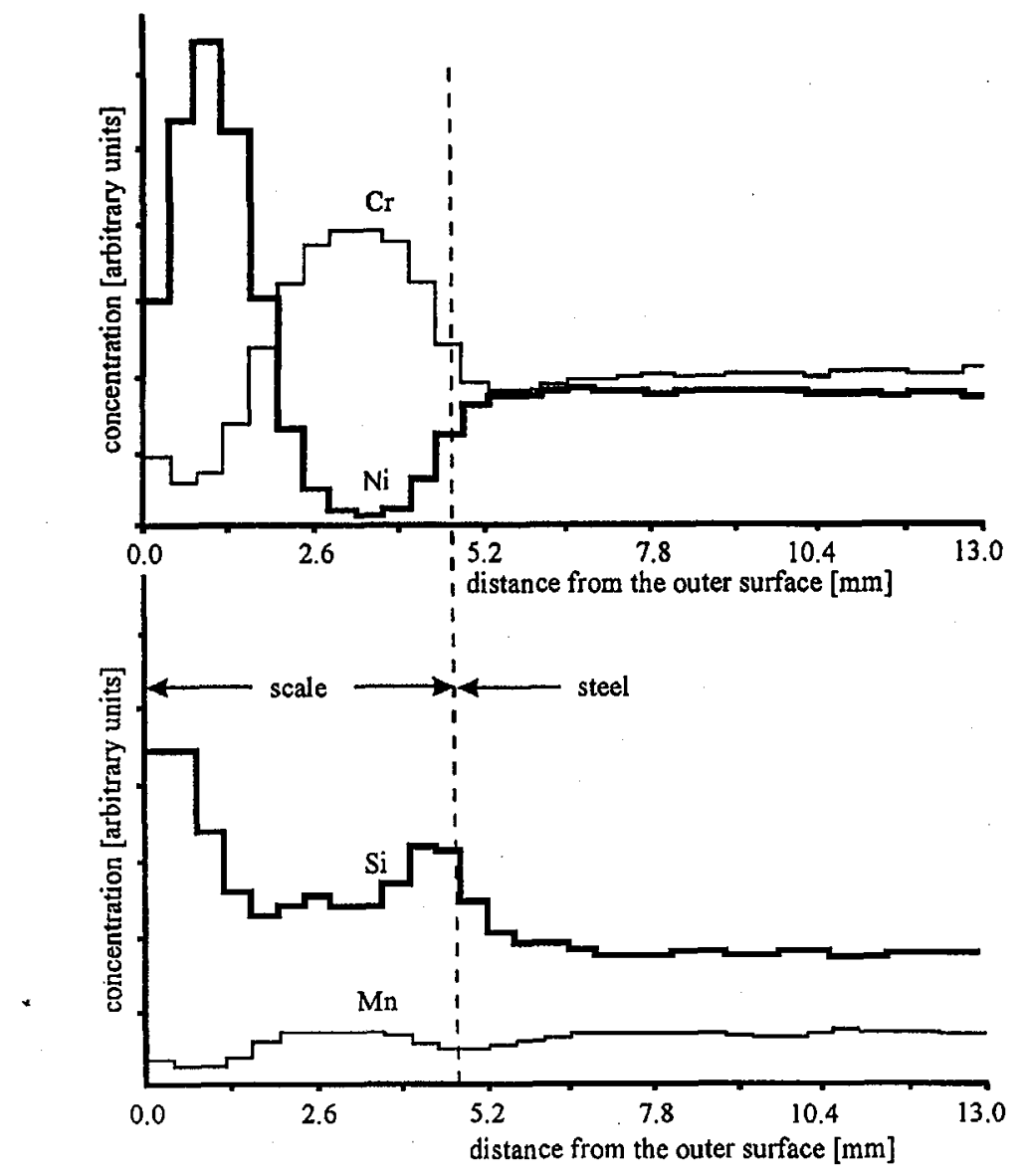

Fig. 7. Variation in the concentration of alloying elements along lines perpendicular to the surface of the specimens.

layers of the scale embody mainly nickel oxides while the inner layers - chromium oxides. This finding is confirmed by the variation in the concentration of alloying. elements along lines perpendicular to the surface of the specimens (Fig. 7). There is a strong concentration of $\mathrm{Ni}$ in the vicinity of the outer surface and a peak of $\mathrm{Cr}$ close to the oxide-substrate interface. The oxide scales also exhibit an increased concentration of Si with two peaks: one at the outer surface and the other at the oxide-substrate interface.

Relatively high roughness and the presence of cracks characterize microstructures typical of the near-surface zone. Selective etching reveals also in this zone the grain boundaries exhibiting a lower resistance to the corrosion. The analysis of the concentration of the alloying elements shows that this is related to chromium depletion. The results of X-ray microprobe mapping demonstrate that the grain boundaries in the substrate close to the oxide scale feature a measurable depletion of $\mathrm{Cr}, \mathrm{Mn}, \mathrm{Si}$ and enrichment of $\mathrm{Ni}$. The concentration of $\mathrm{Cr}$ in the vicinity of the scale drops down to less than $8 \%$ - see Fig. 8. 


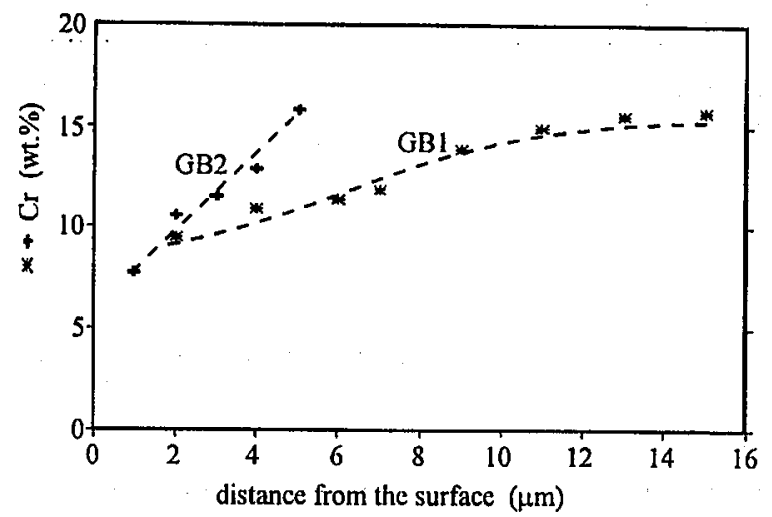

Fig. 8. The concentration of $\mathrm{Cr}$ in the vicinity of the scale along the grain boundaries emerging on the surface of an annealed specimen of austenitic stainless steel.

The results obtained on 316 steel specimens annealed in vacuum and in air can be viewed as composed of three major elements $[12,13]$ :

a) the core which is recrystallized fine-grained austenite with a low density of carbides - this is by far the largest contributing volume and so it will dominate the macroscopic features of the stress-strain curves at larger strains;

b) the near surface zone is associated with a region where both the microchemistry and the microstructure are modified - perhaps a major factor here is that some of the grain boundaries have become sensitized - which suggests that the carbide populations and solute chemistry is altered;

c) the oxide scale consists of many layers of different oxides and it is associated with variable degrees of surface roughness - which in turn will act as stress. concentrators.

Loading the system to a given small strain may be assumed to strain the cylindrical core, the near surface zone "tube" and the oxide scale tube to the same level. In the elastic range, the measured modulus will be based on some form of law of mixtures behavior. One may then assume that the oxide scale will rupture (in a brittle manner) presumably from the outside and these cracks will propagate towards the near-surface zone. It is then the constraints at the scale/near-surface zone interface, which will define how flow occurs and propagates into the material. Serrations on the stress-strain curve suggest that the flow is localized (geometrically representing something like a Luders band) and one may suggest that this is because individual oxide cracks (possibly together with sensitized grain boundaries in the near-surface zone region) act as stress concentrators which encouraged localized flow.

\section{References}

[1] M.F. Ashby, Materials Selection in Mechanical Design, Pergamon Press, Oxford 1992.

[2] G.E. Dieter, Mechanical Metallurgy, McGraw-Hill, London 1988. 
[3] H.G. Van Bueren, Imperfections in Crystals, Interscience Publ., New York 1960.

[4] J.P. Hirlt, J. Lothe, Theory of Dislocations, Wiley Interscience, New York 1982.

[5] N. Hansen, B. Ralph, Acta Metall. 30, 411 (1982).

[6] K.J. Kurzydłowski, R.A. Varin, W. Zielinski, Acta Metall. 32, 71 (1984).

[7] K.J. Kurzydłowski, B. Ralpl, The Quantitative Description of the Microstructure of Materials, CRC Press, Boca Raton 1995.

[8] E.O. Hall, Proc. Phys. Soc. Lond. 643, 747 (1951).

[9] N.J. Petch, J. Iron. Inst. London 173, 25 (1953).

[10] K.J. Kurzydłowski, B. Ralph, J.J. Bucki, A. Garbacz, Mater. Sci. Eng. A 205, 127 (1996).

[11] A.H. Cottrell, B.A. Bilby, Proc. Phys. Soc. Lond. A 62, 49 (1949).

[12] A.A.Adbduluyahed, K. Rożniatowski, K.J. Kurzydłowski, Scr. Metall. Mater. 33, 1489 (1995).

[13] A.A. Abduludyahed, Ph.D. Thesis, Warsaw University of Technology, 1998. 